\title{
Primeiro registro de Mesoclemmys tuberculata (Reptilia, Testudines, Chelidae) em área de Cerrado no Estado de Minas Gerais, Sudeste do Brasil
}

\author{
Adriano Lima Silveira ${ }^{1 *}$ \\ Raquel Valinhas e Valinhas ${ }^{2}$ \\ ${ }^{1}$ Setor de Herpetologia, Departamento de Vertebrados \\ Museu Nacional, Universidade Federal do Rio de Janeiro \\ Quinta da Boa Vista, São Cristóvão, CEP 20940-040, Rio de Janeiro-RJ, Brasil \\ ${ }^{2}$ Laboratório de Zoologia, Centro Universitário de Patos de Minas \\ Rua Major Gote, 808, Caiçaras, CEP 38702-054, Patos de Minas-MG, Brasil \\ *Autor para correspondência \\ biosilveira@yahoo.com.br
}

Submetido em 06/02/2010

Aceito para publicação em 02/08/2010

\section{Resumo}

Mesoclemmys tuberculata é uma espécie de cágado que se distribui no nordeste do Brasil, registrado principalmente na Caatinga e em algumas localidades na Mata Atlântica e no Cerrado. Neste trabalho é reportado o primeiro registro da espécie em área de Cerrado no Estado de Minas Gerais, o qual constitui o único registro conhecido no estado em localidade especificada. Durante amostragem em campo, um espécime de M. tuberculata foi coletado no Município de João Pinheiro, noroeste do estado, em área nuclear do Cerrado na bacia do rio São Francisco. A localidade do presente registro constitui os limites meridional e ocidental da distribuição conhecida de M. tuberculata, assim como, a localidade mais interiorana de registro da espécie no bioma Cerrado.

Unitermos: Cerrado, Chelidae, distribuição geográfica, Mesoclemmys tuberculata, Minas Gerais

\section{Abstract}

First record of Mesoclemmys tuberculata (Reptilia, Testudines, Chelidae) in the Cerrado area of Minas Gerais state, southeastern Brazil. Mesoclemmys tuberculata is a turtle species that is distributed in northeastern Brazil, recorded mainly in the Caatinga and at some localities in the Atlantic Forest and Cerrado. In this paper we report the first species record in an area of Cerrado of Minas Gerais state, and it is the only known state record in a specific location. During field sampling, a specimen of M. tuberculata was collected in the municipality of João Pinheiro, northwest state, in a Cerrado nuclear area in the São Francisco river basin. The locality of this record is the southern and western limits of M. tuberculata's known distribution, as well as the most inland locality of species record in the Cerrado biome.

Key words: Cerrado, Chelidae, geographic distribution, Mesoclemmys tuberculata, Minas Gerais 
O gênero Mesoclemmys Gray, 1863 é atualmente composto por dez espécies distribuídas pela América do Sul, conhecidas popularmente como cágados (Bour e Zaher, 2005). Mesoclemmys tuberculata (Luederwaldt, 1926), foi descrita a partir de dois exemplares procedentes de "Villa Nova (Est. da Bahia)", a qual foi definida como localidade-tipo. Atualmente, esta localidade constitui o Município de Senhor do Bonfim, Estado da Bahia, Brasil (Bour e Pauler, 1987). Luederwaldt (1926) ainda citou um exemplar procedente de Fortaleza, Estado do Ceará.

Alguns trabalhos posteriores apresentaram uma distribuição mais ampla da espécie sem, no entanto, referirem-se a espécimes testemunhos das localidades de registro, além do material-tipo. Assim, Vanzolini et al. (1980) definiu a distribuição geográfica de $M$. tuberculata como nas caatingas e agrestes do Nordeste do Brasil. Segundo Bour e Pauler (1987), a espécie distribui-se nos estados do Ceará, Pernambuco, Bahia e Minas Gerais. De acordo com Iverson (1992), $M$. tuberculata distribui-se no leste do Brasil no rio São Francisco e bacias adjacentes. Este autor apresentou um mapa da distribuição, o qual pontua algumas localidades (sem nomeá-las) ao longo do bioma Caatinga (estados do Ceará e Bahia), no norte da Mata Atlântica (Bahia e divisa entre Alagoas e Sergipe), ecótono Caatinga/ Mata Atlântica (Pernambuco) e duas localidades no Cerrado (Bahia): rio Grande nas proximidades de Barreiras e na região da foz do rio Corrente com o rio São Francisco, proximidades de Sítio do Mato. Bour e Zaher (2005) definiram a distribuição de M. tuberculata como no nordeste brasileiro, correspondendo à Caatinga e apresentaram um mapa baseado nos trabalhos de Bour e Pauler (1987), Iverson (1992) e McCord et al. (2001). Ainda segundo Souza (2005), a distribuição de M. tuberculata estende-se de um semi-árido nordestino interior até a bacia Oriente Atlântico-Nordeste, incluindo bacias do São Francisco e Parnaíba, abrangendo os biomas Caatinga e Mata Atlântica. Esses dois últimos trabalhos não consideraram os registros de $M$. tuberculata no Cerrado previamente apresentados por Iverson (1992).

Registros pontuais posteriores de $M$. tuberculata foram ainda apresentados para ecossistemas costeiros no litoral do Piauí (Loebmann et al., 2006), da Bahia
(ECOA, 2007), do Maranhão (Batistella et al., 2008) e de Alagoas (Santos et al., 2008), e para o sertão da Bahia e de Pernambuco (Santos et al., 2008).

Pritchard e Trebbau (1984) e Bour e Pauler (1987) citaram a ocorrência de $M$. tuberculata em Minas Gerais, mas sem a indicação de localidades mais específicas. Pritchard e Trebbau (1984) mencionaram um exemplar procedente de Minas Gerais e depositado no Museum of Comparative Zoology, Harvard University (MCZ 38682) e o mapa apresentado por Bour e Zaher (2005) abrange aproximadamente todo o bioma Caatinga, incluindo o extremo norte de Minas Gerais. Mas, em ambos os trabalhos, não foram citadas localidades mais precisas de registros e a distribuição apresentada no mapa supracitado pode ser considerada apenas como potencial. Neste contexto, não existem registros publicados de $M$. tuberculata em localidades mais específicas de Minas Gerais.

No presente trabalho é apresentado o primeiro registro de $M$. tuberculata em área de Cerrado no Estado de Minas Gerais, o qual constitui o único registro conhecido no estado em uma localidade mais específica.

Durante amostragens em campo, foi coletado um exemplar fêmea de M. tuberculata (Figura 1) no Município de João Pinheiro (17³0’00"S; 46²7’40”O; $554 \mathrm{~m}$ altitude), noroeste de Minas Gerais (Figura 2), uma área nuclear do Cerrado, na bacia do rio São Francisco. O espécime foi preservado e depositado na Coleção de Répteis do Museu Nacional / Universidade Federal do Rio de Janeiro (MNRJ 18676). A vegetação da área foi caracterizada segundo Ribeiro e Walter (1998).

A região onde $M$. tuberculata foi encontrada em João Pinheiro apresenta um complexo ecossistema com formações de campo limpo, campo cerrado, cerrado sentido restrito e cerradão, abrigando várias lagoas naturais (região conhecida localmente como "campinas"), e situa-se nas proximidades do rio da Prata (afluente do rio Paracatu), cujas margens abrigam formações de mata ciliar e vereda, com grande número de lagoas marginais em meandros (Figura 3). Na região, as lagoas das campinas são, provavelmente, os ambientes mais utilizados por $M$. tuberculata. 


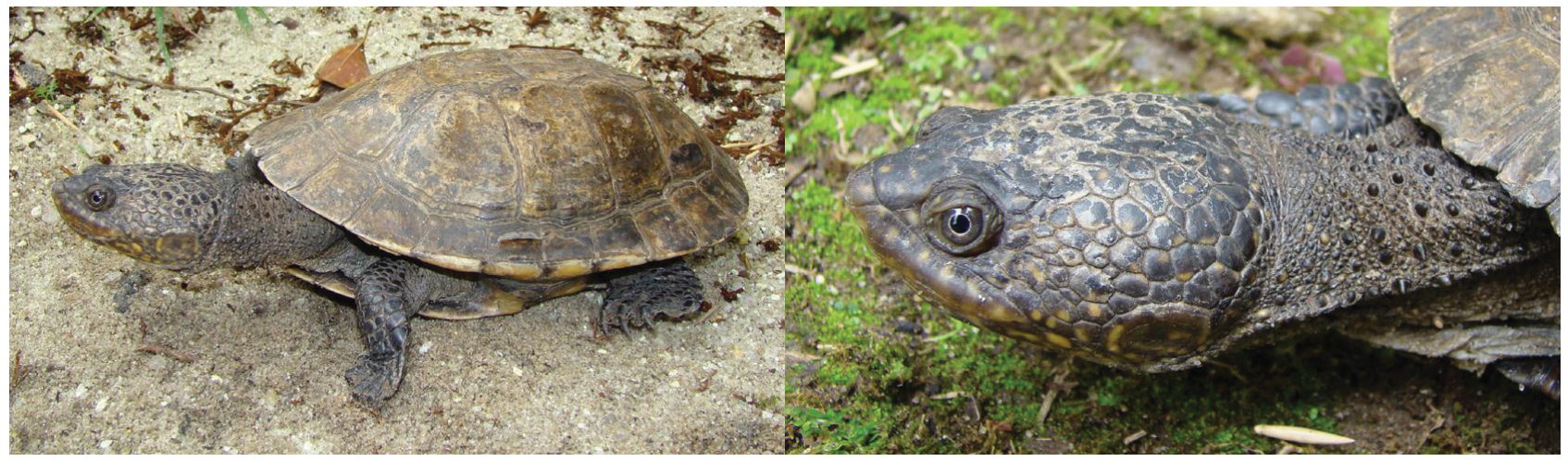

FIGURA 1: Exemplar fêmea de Mesoclemmys tuberculata (MNRJ 18676) coletado no Município de João Pinheiro, Minas Gerais.

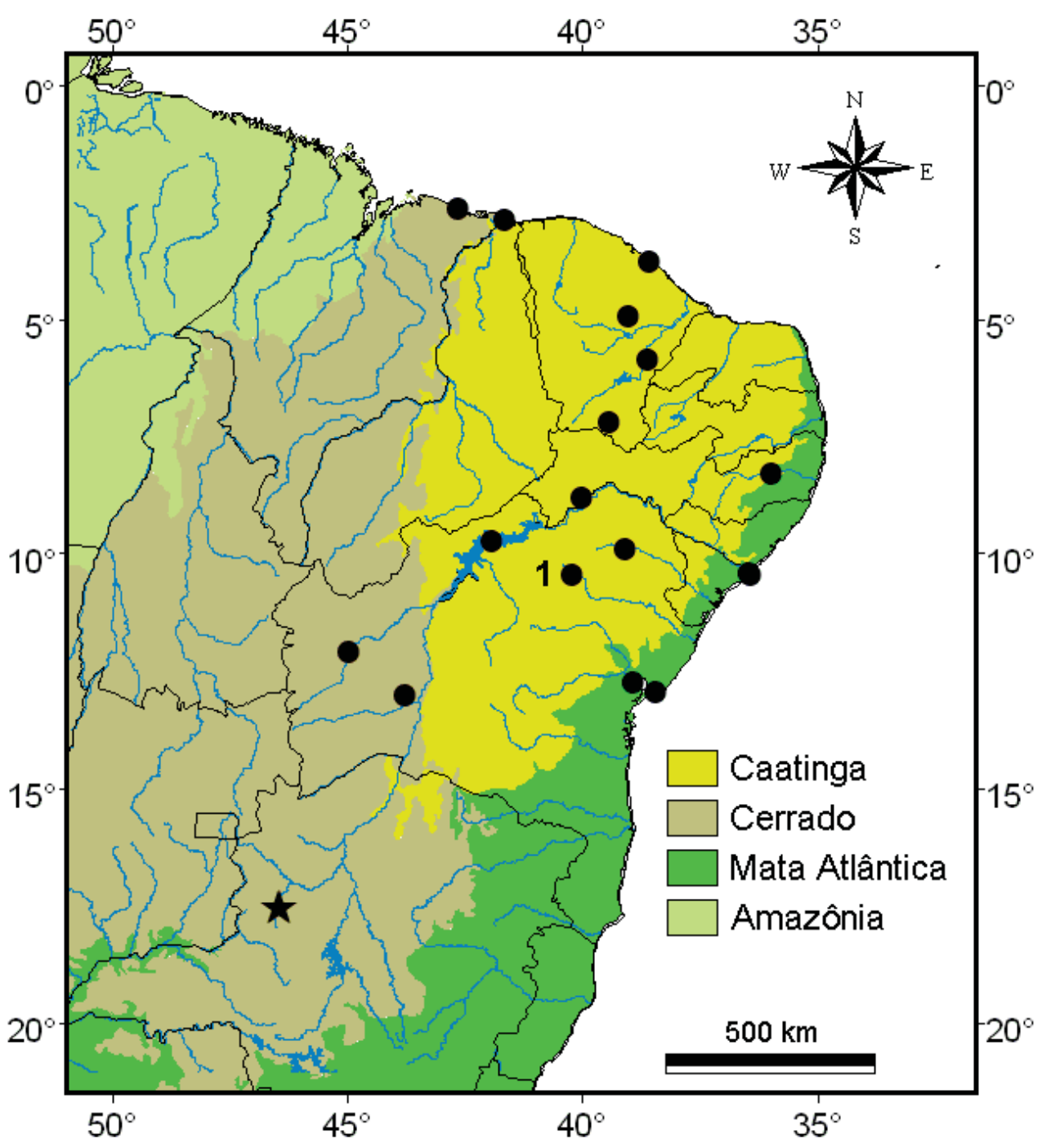

FIGURA 2: Distribuição geográfica de Mesoclemmys tuberculata. Círculos - registros prévios segundo Luederwaldt (1926), Bour e Pauler (1987), Iverson (1992), Loebmann et al. (2006), ECOA (2007), Batistella et al. (2008) e Santos et al. (2008). Estrela novo registro no Município de João Pinheiro, Minas Gerais. 1 - localidade-tipo da espécie. 


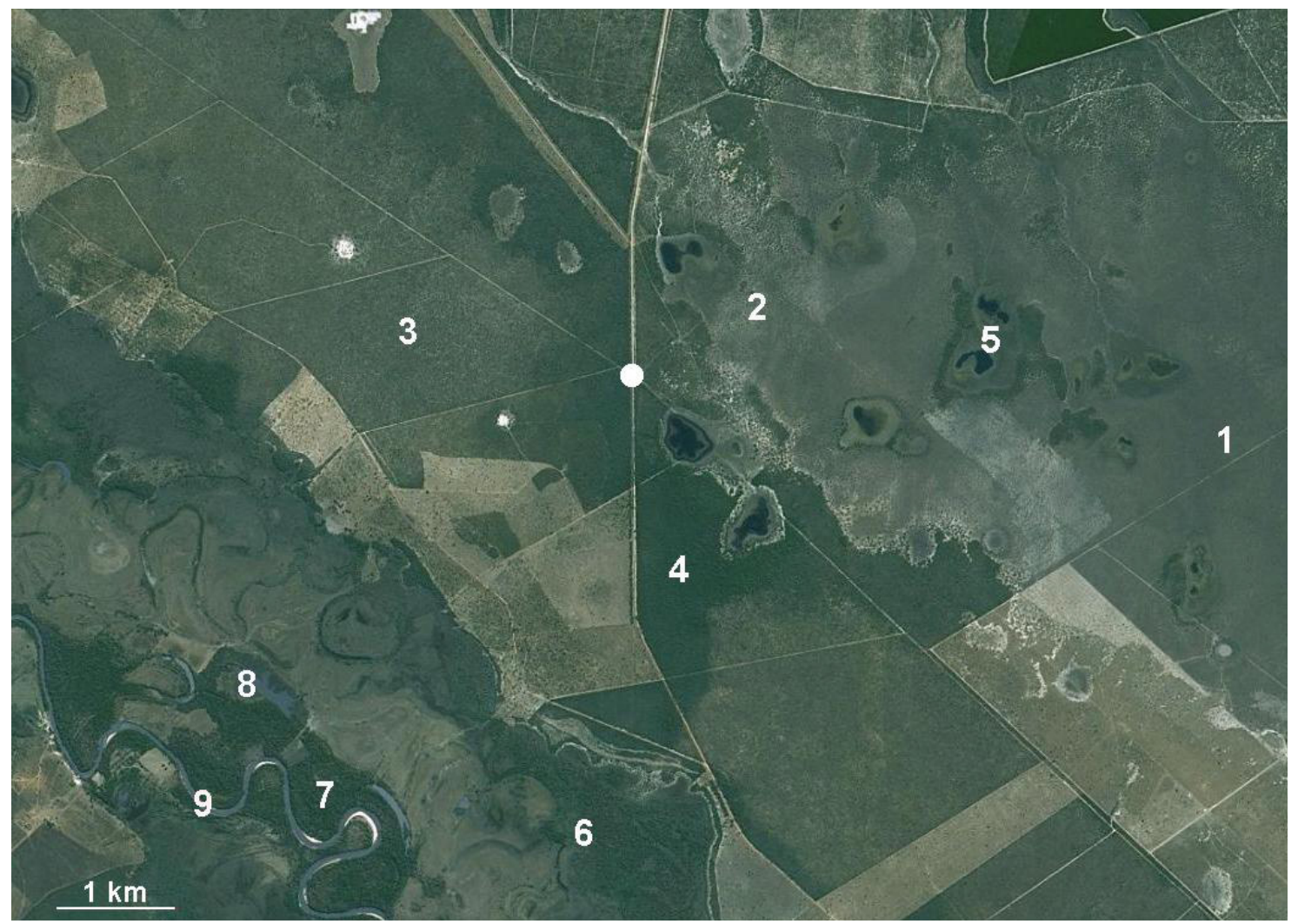

FIGURA 3: Feições do ecossistema da área de registro de Mesoclemmys tuberculata em João Pinheiro, MG. 1 - campo limpo, 2 - campo cerrado, 3 - cerrado sentido restrito, 4 - cerradão, 5 - lagoa de campina, 6 - vereda, 7 - mata ciliar, 8 - lagoa marginal, 9 - rio da Prata, ponto - localidade de coleta do espécime. Foto de satélite retirada do programa Google Earth 2009.

A localidade do presente registro, em João Pinheiro, situa-se a cerca de $570 \mathrm{~km}$ a sudoeste da localidade de registro prévio mais próxima da espécie (região da foz do rio Corrente com o São Francisco, Bahia, segundo Iverson, 1992) e constitui os limites meridional e ocidental da distribuição conhecida de $M$. tuberculata, assim como, a localidade mais interiorana de registro da espécie no Cerrado (Figura 2). Com base neste registro, a ocorrência de $M$. tuberculata passa a ser esperada para demais áreas de Cerrado da bacia do São Francisco no noroeste de Minas Gerais, assim como, para áreas de Caatinga no norte do estado.

Com base em todos os registros aqui apresentados, a distribuição conhecida de $M$. tuberculata pode ser redefinida como ao longo da bacia do rio São Francisco, desde o noroeste de Minas Gerais até sua foz, abrangendo áreas de Cerrado e Caatinga; ao longo do bioma Caatinga; e ao longo da costa atlântica do nordeste do Maranhão até o centro-leste da Bahia, em ecossistemas costeiros; incluindo os estados do
Maranhão, Piauí, Ceará, Pernambuco, Alagoas, Bahia e Minas Gerais, Brasil.

\section{Agradecimentos}

Agradecemos a José Valinhas de Castro e Rita de Cássia Valinhas Costal pelo auxílio na coleta do exemplar e a Elizângela S. Brito, Daniel Loebmann, Alexandre M. Batistella, Henrique B. Ribeiro e Rafael Bernhard pelo fornecimento de literatura especializada.

\section{Referências}

Batistella, A.; Pötter, C.; Barreto, L.; Vogt, R. 2008. Geographic distribution: Mesoclemmys tuberculata. Herpetological Review, 39 (1): 107-108.

Bour, R.; Pauler, I. 1987. Identité de Phrynops vanderhaegei Bour 1973 et des espèces affines. Mésogée, 47: 3-23.

Bour, R.; Zaher, H. 2005. A new species of Mesoclemmys, from the open formations of northeastern Brazil (Chelonii, Chelidae). Papéis Avulsos de Zoologia, 45 (24): 295-311. 
ECOA. 2007. Animais e plantas do Parque Metropolitano de Pituaçu - Lista de espécies. Disponível em $<$ http://www.ucsal.br/ pesquisa/ecoa/pesq_apresentacao.asp $>$. Acesso em 20 de outubro de 2009.

Iverson, J. B. 1992. A revised checklist with distribution maps of the turtles of the World. Privately Printed, Richmond, USA, 363pp.

Loebmann, D.; Mai, A. C. G.; Garcia, A. M. 2006. Reptilia, Chelidae, Mesoclemmys tuberculata: Geographic distribution extension. Check List, 2 (1): 32-33.

Luederwaldt, H. 1926. Os chelonios brasileiros, com a lista das espécies do Museu Paulista. Revista do Museu Paulista, 14: 405479.

McCord, W. P.; Joseph-Ouni, M.; Lamar, W. W. 2001. A taxonomic reevaluation of Phrynops (Testudines: Chelidae) with the description of two new genera and a new species of Batrachemys. Revista de Biología Tropical, 49: 715-764.
Pritchard, P. C. H.; Trebbau, P. 1984. The turtles of Venezuela. Society for the Study of Amphibians and Reptiles, Oxford, UK, 403pp.

Ribeiro, J. F.; Walter, B. M. T. 1998. Fitofisionomias do bioma Cerrado. In: Sano, S. M. \& Almeida, S. P. (Eds). Cerrado: ambiente e flora. EMBRAPA/CPAC, Planaltina, Brasil, p. 87-166.

Santos, F. J. M.; Peña, A. P.; Luz, V. L. F. 2008. Considerações biogeográficas sobre a herpetofauna do submédio e da foz do rio São Francisco, Brasil. Estudos, 35 (1/2): 59-78.

Souza, F. L. 2005. Geographical distribution patterns of South American side-necked turtles (Chelidae), with emphasis on Brazilian species. Revista Española de Herpetología, 19: 33-46.

Vanzolini, P. E.; Ramos-Costa, A. M. M.; Vitt., L. J. 1980. Repteis das Caatingas. Rio de Janeiro, Academia Brasileira de Ciências, $161 \mathrm{pp}$. 